\title{
Jóvenes y trayectorias a la vida adulta. Desigualdades, retos y nuevas formas en un contexto de crisis
}

\author{
MERCEDES ALCAÑIZ y VICENT QUEROL \\ Departament de filosofia I Sociologia Universitat Jaume I. Castelló
}

Resumen

La juventud se ha convertido, desde el inicio de la crisis en 2008, en un tema mediático y objeto de estudio e investigación así como de diseño de políticas públicas que incluyan el objetivo de paliar la situación de dificultad y precariedad en la que se encuentran muchos jóvenes en la actualidad. Jóvenes con una gran formación y que tienen que abandonar el país por no encontrar empleo adecuado en España; jóvenes que abandonaron el sistema educativo para incorporarse al mercado laboral y que con la crisis se han quedado sin empleo; jóvenes que no pueden emanciparse por carecer de medios y que como consecuencia de ello, alargan la permanencia en casa de sus padres. Jóvenes que en definitiva ven un futuro incierto en sus vidas. El objetivo de esta introducción es ofrecer una breve panorámica y una presentación de los diferentes artículos incluidos en el presente número de la revista.

Palabras clave: juventud, transición, emancipación, precariedad.

\begin{abstract}
Youth has become since the beginning of the crisis in 2008, a media subject and also an object of study and research as well as an object of design of public policies with the aim of alleviating the difficult and precarious situation in which many young people are today.Young highly educated who have to leave the country because they don't find suitable employment in Spain; young people who left the education system to enter the labor market and lost their jobs with this crisis; young people who can not emancipate for lack of means and as a result, extend the stay at their parental home. Young ultimately see an uncertain future in their lives. The purpose of this introduction is to provide a brief overview and presentation of the different articles in this issue of the magazine.
\end{abstract}

Keywords: youth, transition, emancipation, precariousness.

El paso de la juventud a la vida adulta ha sido considerado un momento trascendental en las diferentes culturas, siendo ritualizado y simbolizado como expresión del cambio en el ciclo vital de una persona y, a la vez, inicio de una nueva etapa en la vida. 
En las sociedades modernas actuales, el proceso de transición de la juventud a la vida adulta se entiende desde dos puntos de vista: el primero hace referencia al paso del sistema educativo a la vida laboral y el segundo al proceso de emancipación de la familia de origen. En ambos casos se han producido transformaciones importantes que se han visto agudizadas por el actual contexto de crisis en el cual los y las jóvenes forman parte de los colectivos más afectados.

Ahora bien, la transición no mantiene una continuidad y similitud en la diversidad de las sociedades sino que debido a la estructura política, social y familiar, se observan diferencias y divergencias en cuanto a dicho proceso. La existencia de políticas familiares y políticas para la juventud así como las características del mercado laboral y la cultura familista imperante influyen en las diversas modalidades de transición existentes.

En el caso español, se ha producido en los últimos años un doble acontecimiento que explicamos a continuación: por una parte se ha reducido en siete puntos porcentuales el peso del grupo de edad entre 15 y 34 años. Así, en 1991, este grupo etario representaba el 32,2\% en el total de la población mientras que en 2011, la proporción desciende a 25\%. Por otra parte, la emancipación de los jóvenes del hogar familiar es cada vez más tardía agudizándose este comportamiento desde la crisis iniciada en 2008. Según datos del Observatorio de la Emancipación, en 2013 tan solo el 20,9\% de los jóvenes menores de 30 años viven de manera independiente. La proporción de jóvenes emancipados asciende con la edad y en el grupo entre 30-34 años, ya representa el 72,7\%.

Lo expuesto en el párrafo anterior nos permite concluir que los jóvenes son menos que en épocas anteriores pero que viven más tiempo en casa de sus padres. Con el descenso de la fecundidad, el número de criaturas desciende, si bien, están durante más tiempo apegadas al nido familiar.

Por otra parte, se produce otro acontecimiento en la relación formación-empleo que se relaciona con lo dicho más arriba. Este acontecimiento tiene que ver con la ampliación de los años de formación y consecuentemente con la tardanza en incorporarse al mercado laboral.

La juventud actual española se caracteriza por su mayor nivel de estudios ya que se incrementa el número de jóvenes que tienen un título universitario, considerándose la juventud mejor formada de la historia de nuestro país; ahora bien, que tengan estudios universitarios no quiere decir que todos ocupen puestos adecuados al nivel educativo obtenido. En el caso de los jóvenes españoles, el 28,8\% de los jóvenes con estudios universitarios no lo ocupan, siendo el país europeo con la tasa más elevada de sobre-cualificación.Aspecto que acontece más en las jóvenes motivado por 
los problemas que conlleva la crianza de los hijos e hijas, todavía asimétrica entre mujeres y hombres en nuestra sociedad y que perjudica la carrera profesional de muchas mujeres a pesar de que en la actualidad, son más las universitarias que los universitarios.

La consecución de un incremento importante en el número de titulados y tituladas universitarias no evita que según datos de Eurostat (2014), el abandono escolar en España sea el más alto de la Unión Europea. Un 23,5\% de los y las jóvenes españolas abandonan los estudios en algún momento de su trayectoria educativa; si bien, esta proporción ha ido descendiendo desde 2008, año de inicio de la crisis económica.

La crisis económica ha afectado de manera especial a los jóvenes, los cuales se han convertido junto con los inmigrantes y las mujeres en los colectivos más afectados. Su inserción en el mercado laboral se ha retrasado, se ha precarizado y se ha incrementado el grado de incertidumbre respecto de su futuro. El grupo de edad entre 16-19 ha visto como se reducía su tasa de actividad a la mitad desde 2008 tanto para hombres como para mujeres mientras que la tasa de paro ha sido para el último trimestre de 2014 (EPA, 2014) del 62,9\% para los hombres y 70\% para las mujeres. Este grupo etario es, pues, el más afectado por la crisis.

En esta coyuntura de crisis, las mujeres jóvenes entre 25-29 años han incrementado su participación laboral en 8 puntos mientras que las mujeres con edades comprendidas entre 30 y 34 años han incrementado su participación en 17 puntos porcentuales.

En el contexto de incorporación precaria al mercado laboral no podemos obviar que España es, después de Polonia, el país europeo con mayores tasas de temporalidad en el empleo afectando esta situación a los jóvenes de manera especial. Si la tasa de temporalidad es para el conjunto de la población activa de $23,7 \%$ (EPA, $3^{\circ}$ trimestre 2014) para la población entre 16 y 24 años la tasa se eleva al $66,5 \%$ de los contratos. El porcentaje de temporalidad se ha incrementado en casi 11 puntos porcentuales desde 2008.

Para concluir esta breve fotografía sobre la situación de la juventud en España, preciso es aludir al incremento de las jornadas a tiempo parcial: este tipo de jornadas se ha incrementado desde 2008 en 400.000 personas $\mathrm{y}$ afecta en mayor medida a jóvenes y a mujeres. La jornada a tiempo parcial se considera también precaria ya que junto con el hecho de cobrar menos sueldo, se tienen menos posibilidades de promoción y las consecuencias en la jubilación también serán perjudiciales para los o las que se acojan a este tipo de jornada.

La referencia sobre la situación de la juventud española expuesta en los párrafos anteriores no pretende que se considere a la juventud como algo 
homogéneo, como un grupo de edad comprendido entre unos límites marcados por criterios técnicos, administrativos o políticos. Decía Pierre Bourdieu que "La juventud es solo una palabra» en alusión a que es una construcción social, la construcción de un grupo en transición a la vida adulta, heterogéneo en su composición y desigual debido a que cada uno o una de los jóvenes ocupa una posición social mediatizada por su origen familiar, étnico, de género, de clase social y de hábitat diferente condicionándoles, sin duda alguna, en el proceso de transición a la vida adulta, y más todavía en el contexto actual de crisis.

El número de la revista Recerca que presentamos a continuación incluye seis artículos, cinco de ellos resultados de investigaciones empíricas realizadas en un contexto local o regional de Argentina, España o México. Si bien la perspectiva de análisis de los diferentes artículos es diferente así como la delimitación de lo que es «población joven», todos ellos coinciden en la consideración de la inseguridad y vulnerabilidad como las características que mejor definen a la juventud en la actual situación de crisis.

La relación entre juventud y movilidad es tratada en «Fuga de cerebros y biografías low cost: nueva etapa en la precarización juvenil» de Antonio Santos y David Muñoz. Este artículo se centra desde un punto de vista teórico y crítico en la denominada fuga de cerebros o movilidad externa de los jóvenes. Los autores consideran que esta movilidad es un episodio que refleja la precariedad del mercado laboral, relacionándolo con el predominio de la escuela del Capital Humano que considera al trabajador como empresario para sí mismo, hacerse empleable, entendiendo por este concepto la capacidad de producirse a sí mismo, de gestionar su capital humano y su carrera incluida su capacidad de movilidad. Frente a la perspectiva cosmopolita utilizada en otros estudios sobre la juventud los autores de este artículo consideran que la lógica del capital humano está colocando a los jóvenes afectados por la movilidad laboral en una situación de riesgo y vulnerabilidad crecientes.

Alessandro Gentile nos presenta en «Jóvenes titulados superiores en la encrucijada de la crisis» los resultados de las entrevistas realizadas a 20 jóvenes españoles titulados universitarios entre los 24 y los 30 años y que en la actualidad están trabajando con contratos precarios con el objetivo de conocer cómo la coyuntura actual influye en sus posibilidades de independencia. El análisis de los discursos de los entrevistados y entrevistadas se lleva a cabo desde tres perspectivas interpretativas: la crisis como trampa, la crisis como obstáculo y la crisis como oportunidad. La conclusión a la que llega Gentile es que en la actualidad se produce una fragmentación 
de las transiciones a la vida adulta muy compleja al acentuarse agudamente las diversidades adscritas individual y socialmente.

Continuando en el ámbito universitario, Guillermina Díaz, Natalia Ix-chel Vázquez y Araceli Pérez Damián , escriben «La sombra de la violencia estructural en los jóvenes universitarios». Estas autoras tienen en cuenta la definición de Johan Galtung sobre violencia estructural aludiendo en su artículo que este tipo de violencia se evidencia en la sociedad cuando existe incapacidad por parte de los gobiernos para cubrir las necesidades básicas de la población para aplicar esta perspectiva teórica en su investigación para constatar el nivel de violencia estructural existente en los y las jóvenes egresadas de la universidad en el Estado de México. La investigación se realizó utilizando la técnica de grupos focales a jóvenes entre 21-26 años con el objetivo de conocer si los y las jóvenes participantes eran conscientes, y percibían, la violencia estructural de la que son objeto en su transición a la vida adulta, en el paso de la etapa estudiantil a la etapa laboral.

El artículo «Las trayectorias de vida de los jóvenes de Santa Cruz de Tenerife en tiempos de crisis: una aproximación cualitativa» presentado por Gomer Betancor Nuez y Josué Gutiérrez Barroso, forma parte de la investigación realizada en Santa Cruz de Tenerife Perspectivas de futuro de los jóvenes de Santa Cruz de Tenerife: nuevos retos, nuevas realidades. La investigación tiene como objetivo analizar las perspectivas juveniles acerca de la realidad social, política y económica que marcan las diferentes trayectorias de vida de la juventud. La metodología utilizada ha sido cualitativa, mediante la realización de 5 grupos de discusión a jóvenes entre 18 y 29 años. Los resultados apuntados por los autores resaltan las pocas expectativas de futuro que ven en Santa Cruz, sobre todo a corto y medio plazo, y que influyen en la decisión de emigrar bien a la Península Ibérica o bien al extranjero para encontrar un futuro mejor.

Sara Moreno en "Análisis de las transiciones juveniles desde la perspectiva de género: entre la influencia del ciclo vital y el cambio generacional» analiza cómo el género condiciona las oportunidades de los jóvenes durante la etapa del ciclo de vida que va desde la dependencia de la niñez a la autonomía de la vida adulta. Para ello se basa en los datos de la Encuesta de la Juventud de Catalunya (2012), realizada a jóvenes entre 15 y 34 años residiendo en Cataluña. La conclusión apuntada por la autora es que de los tres tipos de transiciones contempladas (la educativa, la laboral y la familiar) solo existe igualdad de oportunidades en la transición educativa persistiendo la desigualdad en el resto de transiciones a la vida adulta.

Finamente, Analía Otero presenta en su artículo «La otra cara. Políticas públicas, inclusión laboral y jóvenes urbanos de sectores populares en un 
movimiento social», una reflexión sobre las políticas públicas implementadas desde el Gobierno Nacional de Argentina hacia los movimientos sociales. Se analiza en concreto el impacto del Programa social con trabajo (PAT) en el Frente Popular Darío Santillán (FPDS) ubicado en Lanús, localidad del primer cordón industrial al sur de la provincia de Buenos Aires. La investigación se llevó a cabo mediante la realización de entrevistas individuales semi-estructuradas. Las conclusiones aludían a que pese a que el Programa no parecía revertir totalmente la situación de los jóvenes, ni alterando sustantivamente su situación económica, sí que se observa que ha estimulado la generación de lazos sociales y vínculos afectivos entre los compañeros del movimiento social. 